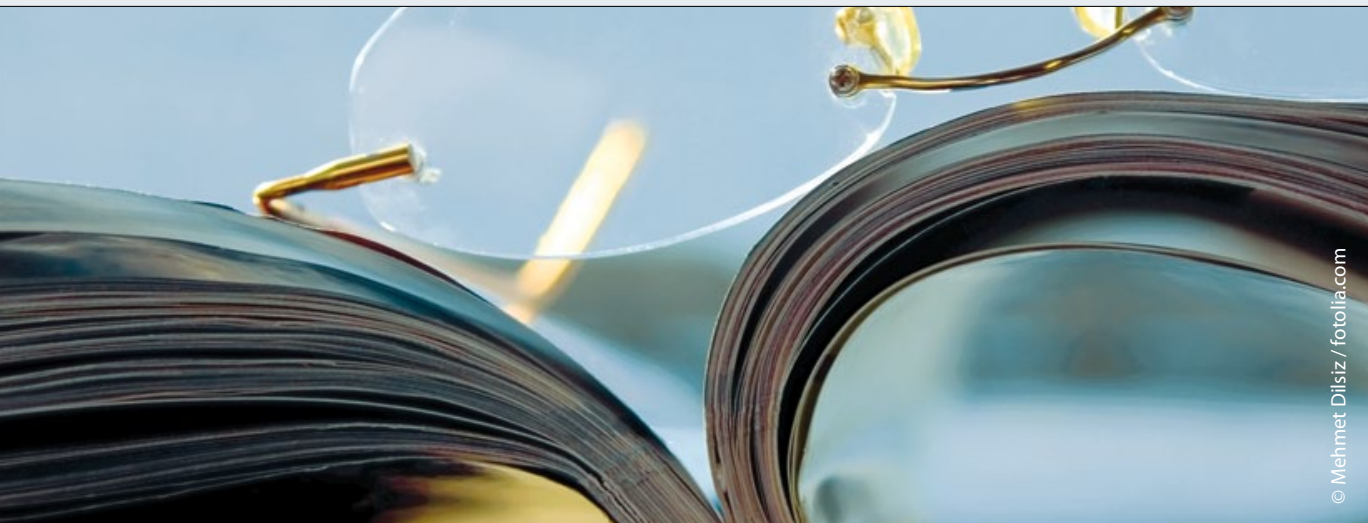

\section{Prostatakarzinom: Hormonstopp mindert Müdigkeit}

Der neue Enzyminhibitor Arbirateronacetat hemmt die Androgen-Biosynthese. Könnte er daher beim kastrationsresistenten, metastasierten Prostatakarzinom auch die mit dieser Erkrankung häufig einhergehende Fatigue mindern?

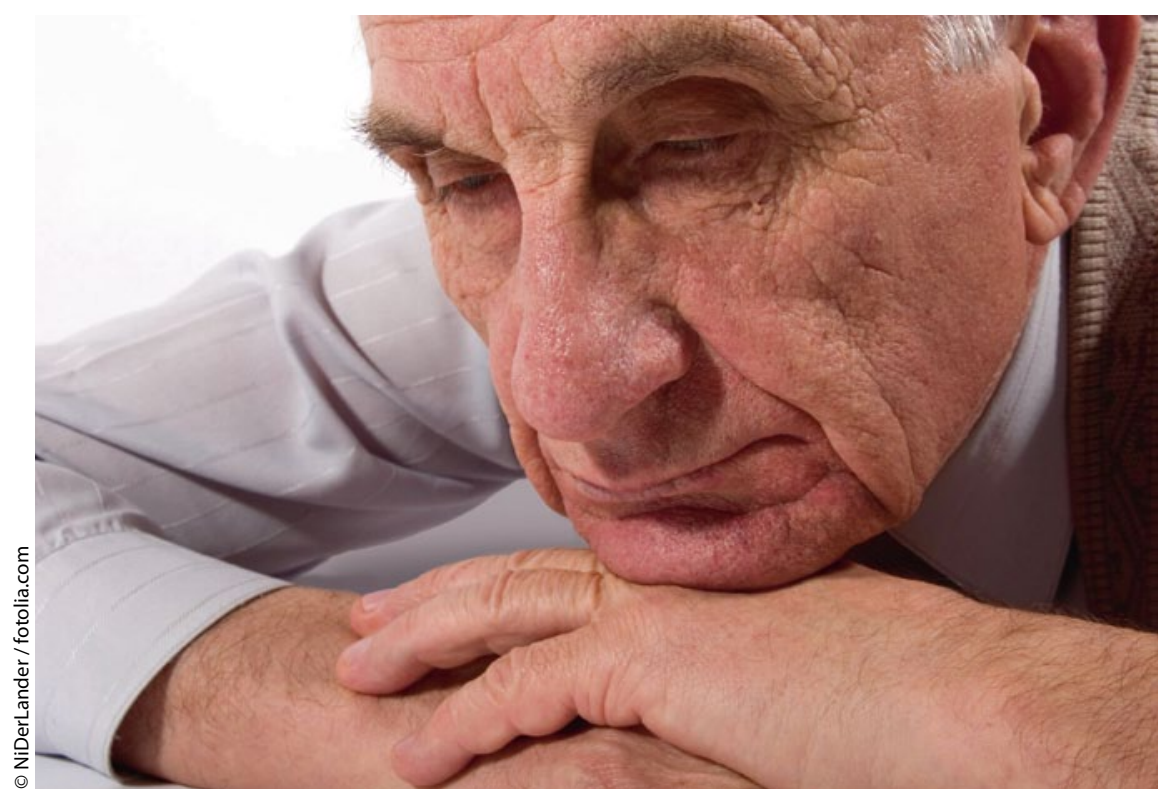

Die tumorbedingte Fatigue macht vielen Prostatakarzinompatienten zu schaffen.

F ür Patienten mit metastasiertem, kastrationsresistentem Prostatakarzinom ist Fatigue eine häufige Erscheinung. Sie ist zum Teil Folge der Erkrankung selbst, eine Nebenwirkung der Therapien oder resultiert aus einer reaktiven Depression. In jedem Fall mindern die schwere Müdigkeit und Kraftlosigkeit die Lebensqualität. Bei fortgeschrittener Krankheit gibt es bisher keine therapeutische Option gegen die Fatigue. Der Enzyminhibitor
Abirateronacetat aber könnte diese Lücke schließen. Zumindest hemmt das Medikament das Tumorwachstum, indem es in den Nebennieren und in der Tumorumgebung die Synthese von Testosteron und anderen Androgenen hemmt, die bei kastrationsresistenten Erkrankungen das Wachstum befeuern.

In einer Phase-III-Studie wurde nun Abirateronacetat plus Prednison mit Placebo plus Prednison im Anschluss an eine
Docetaxel-basierte Chemotherapie verglichen. Nach Radomisierung erhielten 797 Patienten Arbirateronacetet plus Prednison, 398 Patienten kamen in die Vergleichsgruppe. Bei allen Patienten lag zu Beginn der Behandlung eine klinisch signifikante Fatigue vor. Es handelt sich um die erste Phase-III-Studie beim fortgeschrittenen Prostatakarzinom, bei der das Ausmaß der Fatigue mit einem validierten, Fatigue-spezifischen Instrument gemessen wurde, dem Brief Fatigue Inventory. Dieser Fragebogen bestimmt die von den Patienten berichtete Intensität der Fatigue, aber auch, inwieweit die Symptome die Aktivitäten des täglichen Lebens beeinträchtigen.

Im Vergleich zur Kontrollbehandlung erhöhte die Kombinationstherapie signifikant den Anteil der Patienten, die von einer Verbesserung ihrer Fatigue-Intensität berichteten $(58,1 \%$ versus $40,3 \%)$. Auch verbesserte sich die Fatigue-Intensität im Mittel schneller (59 Tage gegenüber 194 Tage), und der ungünstige Einfluss der Fatigue auf das tägliche Leben wurde geringer (55\% versus $38 \%$ ).

Fazit: Bei Patienten, die nach einer Docetaxel-basierten Chemotherapie eine Progress haben, bessert sich durch die Kombination aus Abirateronacetat und Prednison die von den Patienten selbst berichtete Fatigue-Intensität im Vergleich zu Prednison plus Placebo. Christina Berndt

Sternberg $\mathrm{CN}$ et al. Effect of abiraterone acetate on fatigue in patients with metastatic castration-resistant prostate cancer after docetaxel chemotherapy. Ann Oncol 2013; 24: $1017-25$ 